\title{
A note on the second kind q-Apostol Bernoulli numbers, polynomials, and Zeta function
}

\author{
C. K. An, H. Y. Lee*, Y. R. Kim \\ Department of Mathematics, Hannam University, Daejeon 306-791, Korea.
}

\begin{abstract}
In this paper we consider a new type of the q-Apostol Bernoulli numbers and polynomials. Firstly, we define the q-Apostol Bernoulli numbers and polynomials by making use of their generating function. Also, we observe many properties, i.e., the recurrence formula, the difference equation, the differential relation.
\end{abstract}

Keywords: The second kind q-Apostol Bernoulli polynomials, the second kind q-Apostol Bernoulli numbers, zeta function. 2010 MSC: 05A30, 11M35, 11B83.

(C)2019 All rights reserved.

\section{Introduction}

Bernoulli numbers were discovered by Jacob Bernoulli in the 17th century. As it is well known, Bernoulli numbers are related to many important properties appearing in mathematics and physics. Thereby many mathematicians have studied the Bernoulli numbers and polynomials. In this paper, we introduce the generalized second kind Bernoulli numbers and polynomials and derive many interesting properties. In the last section, we will give a relation between the $B_{n, q}(x, \lambda)$ and $E_{n, q}(x, \lambda)$.

Throughout this paper, we will use the following notation: $\mathbb{N}$ denotes the set of natural numbers, $\mathbb{Z}$ denotes the ring of rational integers, $\mathbb{Q}$ denotes the field of rational numbers, $\mathbb{C}$ denotes the set of complex numbers, $\mathbb{Z}_{0}^{+}=\mathbb{N} \cup\{0\}$, and $\mathbb{Z}_{0}^{-}=\{0,-1,-2, \cdots\}$. In this paper, $[x]_{\mathrm{q}}$ is defined as below:

$$
[x]_{\mathrm{q}}=\frac{1-\mathrm{q}^{\mathrm{x}}}{1-\mathrm{q}}, \quad[\mathrm{x}]_{-\mathrm{q}}=\frac{1-(-\mathrm{q})^{\mathrm{x}}}{1+\mathrm{q}} .
$$

And $[x]_{\mathrm{q}}$ has useful properties as following:

$$
[x+y]_{q}=[x]_{q}+q^{x}[y]_{q} .
$$

Note that $[x]_{q}$ tends to $x$ as $q \rightarrow 1$, so $\lim _{q \rightarrow 1}[x]_{q}=x$.

\footnotetext{
*Corresponding author

Email addresses: ack7165@gmail.com (C. K. An), normaliz@hnu.kr (H. Y. Lee), cantor73@naver.com (Y. R. Kim)

doi: $10.22436 /$ jnsa.012.01.06
} 
As a well known definition, the Bernoulli polynomials $B_{n}(x)$ is defined by the following generating function (see, for details, [11], [3, pp.48-49], and [7, pp.25-32]):

$$
\frac{t e^{x t}}{e^{t}-1}=\sum_{n=0}^{\infty} B_{n}(x) \frac{t^{n}}{n !}, \quad|t|<2 \pi
$$

If $x=0$, then $B_{n}=B_{n}(0)$ is called the Bernoulli numbers.

The second kind Apostol Bernoulli polynomials $B_{n}(\lambda)$ have been studied by Lee et al. in 2011 and defined as below (see, $[6,9])$ :

$$
F_{\lambda}(x, t)=\frac{t e^{t}}{\lambda e^{2 t}-1} e^{x t}=\sum_{n=0}^{\infty} B_{n}(x, \lambda) \frac{t^{n}}{n !}, \quad|2 t+\log \lambda|<2 \pi .
$$

If $x=0$, then $B_{n}(\lambda)=B_{n}(0, \lambda)$ is called the second kind Apostol Bernoulli numbers.

For the complex numbers $\lambda \in \mathbb{C}$, the above generating function $F_{\lambda}(x, t)$ can be rewrited as below:

$$
F_{\lambda}(x, t)=\frac{t e^{t}}{\lambda e^{2 t}-1} e^{x t}=-t \sum_{n=0}^{\infty} \lambda^{n} e^{(2 n+1+x) t}, \quad|2 t+\log \lambda|<2 \pi .
$$

The classical Euler polynomials $E_{n}(x)$ are defined by means of the following generating functions (see, $[8,11])$ :

$$
\frac{2}{e^{t}+1} e^{x t}=\sum_{n=0}^{\infty} E_{n}(x) \frac{t^{n}}{n !}, \quad|t|<\pi .
$$

If $x=0$, then $E_{n}=E_{n}(0)$ is called the classical Euler numbers. The second kind Apostol Euler polynomials $E_{n}(x, \lambda)$ are defined by means of the generating function:

$$
\frac{2 e^{t}}{\lambda e^{2 t}+1} e^{x t}=\sum_{n=0}^{\infty} E_{n}(x, \lambda) \frac{t^{n}}{n !}, \quad|2 t+\log \lambda|<\pi .
$$

If $x=0, E_{n}(\lambda)=E_{n}(0, \lambda)$ we called Apostol Euler numbers. Note that, $E_{n}(x)=E_{n}(x, 1)$ and $E_{n}(\lambda)=$ $\mathrm{E}_{\mathrm{n}}(0, \lambda)$.

For the complex numbers $\lambda \in \mathbb{C}$ the above generating function $F_{\lambda}(x, t)$ can be rewrited as below:

$$
\frac{2 e^{t}}{\lambda e^{2 t}+1} e^{x t}=2 \sum_{n=0}^{\infty}(-\lambda)^{n} e^{(2 n+1+x) t}, \quad|2 t+\log \lambda|<\pi .
$$

In Section 2, we introduce the second kind q-Apostol Bernoulli numbers $B_{n, q}(\lambda)$ and polynomials $B_{n, q}(x, \lambda)$.

\section{Definition for the q-Apostol Bernoulli numbers and polynomials and its basic properties}

Definition 2.1. For $\lambda \in \mathbb{C}$ and $|q|<1$, the $q$-Apostol Bernoulli numbers $B_{n, q}(\lambda)$ and polynomials $B_{n, q}(x, \lambda)$ are defined by means of the generaing functions:

$$
\begin{gathered}
F_{\lambda, q}(t)=-t \sum_{n=0}^{\infty} \lambda^{n} e^{[2 n+1]_{q} t}=\sum_{n=0}^{\infty} B_{n, q}(\lambda) \frac{t^{n}}{n !} \\
F_{\lambda, q}(x, t)=-t \sum_{n=0}^{\infty} \lambda^{n} e^{[2 n+1+x]_{q} t}=\sum_{n=0}^{\infty} B_{n, q}(x, \lambda) \frac{t^{n}}{n !} .
\end{gathered}
$$


Definition 2.2. For $\lambda \in \mathbb{C}$ and $|q|<1$, the $q$-Apostol Euler numbers $E_{n, q}(\lambda)$ and polynomials $E_{n, q}(x, \lambda)$ are defined by means of the generaing functions:

$$
2 \sum_{n=0}^{\infty}(-\lambda)^{n} e^{[2 n+1]_{q} t}=\sum_{n=0}^{\infty} E_{n, q}(\lambda) \frac{t^{n}}{n !}, \quad 2 \sum_{n=0}^{\infty}(-\lambda)^{n} e^{[2 n+1+x]_{q} t}=\sum_{n=0}^{\infty} E_{n, q}(x, \lambda) \frac{t^{n}}{n !} .
$$

From Definition 2.1, we can easily get as below:

$$
\sum_{n=0}^{\infty} B_{n, q}(x, \lambda) \frac{t^{n-1}}{n !}=-\sum_{n=0}^{\infty} \lambda^{n} e^{[2 n+1+x]_{q} t} .
$$

Setting $B_{0, q}(x, \lambda)=0$ in the left-hand side of the above equation (2.3), we get the following results.

$$
\sum_{n=0}^{\infty} B_{n, q}(x, \lambda) \frac{t^{n}}{n !}=\sum_{m=0}^{\infty} \frac{1}{m+1} B_{m+1, q}(x, \lambda) \frac{t^{m}}{m !} .
$$

Using binomial theorem in the right-hand side of the above equation (2.3), we get the following results.

$$
\begin{aligned}
-\sum_{n=0}^{\infty} \lambda^{n} e^{[2 n+1+x]_{q} t} & =-\sum_{n=0}^{\infty} \lambda^{n} \sum_{m=0}^{\infty}[2 n+1+x]_{q}^{m} \frac{t^{m}}{m !} \\
& =-\sum_{m=0}^{\infty}\left(\frac{1}{(1-q)^{m}} \sum_{l=0}^{n}\left(\begin{array}{c}
m \\
l
\end{array}\right)(-1)^{l} q^{(x+1) l} \frac{1}{1-\lambda q^{2 l}}\right) \frac{t^{m}}{m !} .
\end{aligned}
$$

Comparing the coefficient of $\frac{\mathrm{t}^{\mathrm{m}}}{\mathrm{m} !}$ on both sides of (2.4) and (2.5), respectivelty, we obtain the Theorem 2.3.

Theorem 2.3. For a nonnegative integer $\mathrm{n}$,

$$
B_{n, q}(x, \lambda)=-\frac{n}{(1-q)^{n-1}} \sum_{l=0}^{n-1}\left(\begin{array}{c}
n-1 \\
l
\end{array}\right) \frac{(-1)^{l}}{1-\lambda q^{2 l}} q^{(x+1) l}, \quad B_{n, q}(\lambda)=-\frac{n}{(1-q)^{n-1}} \sum_{l=0}^{n-1}\left(\begin{array}{c}
n-1 \\
l
\end{array}\right) \frac{(-1)^{l} q^{l}}{1-\lambda q^{2 l}} .
$$

Using Definitions 2.1 and (1.1), we get the following:

$$
\sum_{n=0}^{\infty} B_{n, q}(x, \lambda) \frac{t^{n}}{n !}=-t \sum_{n=0}^{\infty} \lambda^{n} e^{[2 n+1+x]_{q} t}=\sum_{n=0}^{\infty}\left(\sum_{k=0}^{n}\left(\begin{array}{l}
n \\
k
\end{array}\right) B_{k, q}(\lambda) q^{(k-1) x}[x]_{q}^{n-k}\right) \frac{t^{n}}{n !} .
$$

Comparing the coefficient of $\frac{\mathrm{t}^{\mathrm{n}}}{\mathrm{n} !}$ on both sides of (2.6), we obtain the Theorem 2.4.

Theorem 2.4. For a nonnegative integer $n$,

$$
B_{n, q}(x, \lambda)=\sum_{k=0}^{n}\left(\begin{array}{l}
n \\
k
\end{array}\right) B_{k, q}(\lambda) q^{(k-1) x}[x]_{q}^{n-k}
$$

From Definition 2.1, we get the following:

$$
\begin{aligned}
\sum_{n=0}^{\infty} \lambda B_{n, q}(x+2, \lambda)-B_{n, q}(x, \lambda) \frac{t^{n}}{n !} & =-t\left(\sum_{n=0}^{\infty} \lambda^{n+1} e^{[2 n+1+x+2]_{q} t}-\sum_{n=0}^{\infty} \lambda^{n} e^{[2 n+1+x]_{q} t}\right) \\
& =\sum_{n=0}^{\infty} n[x+1]_{q}^{n-1} \frac{t^{n}}{n !}
\end{aligned}
$$

Comparing the coefficient of $\frac{\mathrm{t}^{\mathrm{n}}}{\mathrm{n} !}$ on both sides of (2.7), we obtain the Theorem 2.5. 
Theorem 2.5. For a nonnegative integer $n$,

$$
\lambda B_{n, q}(x+2, \lambda)-B_{n, q}(x, \lambda)=n[x+1]_{q}^{n-1} .
$$

Using result of the Theorem 2.4 to differentiate both sides, we obtain the following:

$$
\frac{\partial}{\partial x}\left(\sum_{n=0}^{\infty} B_{n, q}(x, \lambda) \frac{t^{n}}{n !}\right)=\frac{\partial}{\partial x}\left(-t \sum_{n=0}^{\infty} \lambda^{n} e^{[2 n+1+x]_{q} t}\right)=\frac{q^{x+1}}{q-1} \log q \sum_{n=0}^{\infty} n B_{n-1, q}\left(x, \lambda q^{2}\right) \frac{t^{n}}{n !} .
$$

Comparing the coefficient of $\frac{t^{n}}{n !}$ on both sides of (2.8) we obtain the Theorem 2.6.

Theorem 2.6. For a nonnegative integer $n$,

$$
\frac{\partial}{\partial x} B_{n, q}(x, \lambda)=\frac{q^{x+1}}{q-1} \log q \cdot n \cdot B_{n-1, q}\left(x, \lambda q^{2}\right) .
$$

Integrating both sides using the result of Theorem 2.6, we obtain the following:

$$
\int_{a}^{b} \frac{\partial}{\partial x} B_{n, q}(x, \lambda)=\int_{a}^{b} \frac{q^{x+1}}{q-1} \log q \cdot n \cdot B_{n-1, q}\left(x, \lambda q^{2}\right) d x .
$$

The following equation can be obtained by summarizing the above equation

$$
\frac{q-1}{n \log q}\left(B_{n, q}(b, \lambda)-B_{n, q}(a, \lambda)\right)=\int_{a}^{b} q^{x+1} B_{n-1, q}\left(x, \lambda q^{2}\right) d x .
$$

Thus, the result of the Theorem 2.7 can be obtained.

Theorem 2.7. For a nonnegative integer $n$,

$$
\int_{a}^{b} q^{x+1} B_{n, q}\left(x, \lambda q^{2}\right) d x=\frac{(q-1)\left(B_{n+1, q}(b, \lambda)-B_{n+1, q}(a, \lambda)\right)}{(n+1) \log q} .
$$

From Definition 2.1, we obtain the following:

$$
\begin{aligned}
\sum_{n=0}^{\infty} B_{n, q}(x+y, \lambda) \frac{t^{n}}{n !} & =-t \sum_{n=0}^{\infty} \lambda^{n} e^{[2 n+1+x+y]_{q} t} \\
& =q^{-y} \sum_{n=0}^{\infty} B_{n, q}(x, \lambda) q^{y n} \frac{t^{n}}{n !} \sum_{n=0}^{\infty}[y]_{q}^{n} \frac{t^{n}}{n !} \\
& =\sum_{n=0}^{\infty}\left(\sum_{k=0}^{n}\left(\begin{array}{l}
n \\
k
\end{array}\right) B_{k, q}(x, \lambda) q^{(k-1) y}[y]_{q}^{n-k}\right) \frac{t^{n}}{n !}
\end{aligned}
$$

Comparing the coefficient of $\frac{\mathrm{t}^{\mathrm{n}}}{\mathrm{n} !}$ on both sides of (2.9), we obtain the result of Theorem 2.8.

Theorem 2.8. For a nonnegative integer $n$,

$$
B_{n, q}(x+y, \lambda)=\sum_{k=0}^{n}\left(\begin{array}{l}
n \\
k
\end{array}\right) B_{k, q}(x, \lambda) q^{(k-1) y}[y]_{q}^{n-k} .
$$

From Theorem 2.3, we get the following:

$$
B_{n, q^{-1}}\left(x, \lambda^{-1}\right)=-\frac{n}{(1-q)^{n-1}} \sum_{l=0}^{n-1}\left(\begin{array}{c}
n-1 \\
l
\end{array}\right) \frac{(-1)^{l}}{1-\lambda q^{2 l}} q^{(x+1) l}=(-1)^{n} \lambda q^{n-1} B_{n, q}(-x, \lambda) .
$$

Then we can easily get the following results. 
Theorem 2.9. For a nonnegative integer $n$,

$$
B_{n, q}(x, \lambda)=\frac{(-1)^{n}}{\lambda q^{n-1}} B_{n, q^{-1}}\left(-x, \lambda^{-1}\right) .
$$

From the Definition 2.1, we get

$$
\begin{aligned}
\sum_{n=0}^{\infty}\left\{B_{n, q}(x, \lambda)-\lambda^{k+1} B_{n, q}(x+2 k+2, \lambda)\right\} \frac{t^{n}}{n !} & =-t \sum_{l=0}^{k} \lambda^{l} e^{[x+2 l+1]_{q} t} \\
& =-\sum_{n=1}^{\infty}\left(\sum_{l=0}^{k} n \lambda^{l}[x+2 l+1]_{q}{ }^{n-1}\right) \frac{t^{n}}{n !} .
\end{aligned}
$$

Comparing the coefficient of $\frac{\mathrm{t}^{\mathrm{n}}}{\mathrm{n} !}$ on both sides of (2.10), we obtain the result of Theorem 2.10.

Theorem 2.10. For a nonnegative integer $n$,

$$
\frac{\lambda^{k+1} B_{n, q}(x+2 k+2, \lambda)-B_{n, q}(x, \lambda)}{n}=\sum_{l=0}^{k} \lambda^{l}[x+2 l+1]_{q}^{n-1} .
$$

Remark 2.11. When $\mathrm{q} \rightarrow 1^{-}$, then Theorems 2.3-2.10 become the corresponding properties for the ApostolBernoulli polynomials.

Remark 2.12. When $\lambda \rightarrow 1^{-}$, then equations in Theorems 2.3-2.10 become corresponding properties for the q-Bernoulli polynomials. Setting $\mathrm{q} \rightarrow 1^{-1}$ and $\lambda \rightarrow 1$ in Theorems 2.3-2.10, these formulas become well-known formulas (see [10, pp.532-533]).

\section{The generated function and applications}

By the equations (2.1) and (2.2), we get

$$
\begin{aligned}
\sum_{n=0}^{\infty} B_{n, q}(x, \lambda) \frac{t^{n}}{n !} & =-t \sum_{n=0}^{\infty} \lambda^{n} e^{[2 n+1+x]_{q} t} \\
& =-t e^{\frac{t}{1-q}} \sum_{n=0}^{\infty} \lambda^{n} \sum_{k=0}^{\infty}\left(-\frac{q^{2 n+1+x}}{1-q}\right)^{k} \frac{t^{k}}{k !} \\
& =-t e^{\frac{t}{1-q}} \sum_{k=0}^{\infty} \frac{(-1)^{k} q^{(x+1) k}}{1-\lambda q^{2 k}} \frac{1}{(1-q)^{k}} \frac{t^{k}}{k !}
\end{aligned}
$$

Therefore, we obtain the generating function for $B_{n, q}(x, \lambda)$ as follows:

$$
\sum_{n=0}^{\infty} B_{n, q}(x, \lambda) \frac{t^{n}}{n !}=-t e^{\frac{t}{1-q}} \sum_{n=0}^{\infty} \frac{(-1)^{n} q^{(x+1) n}}{1-\lambda q^{2 n}}\left(\frac{1}{1-q}\right)^{n} \frac{t^{n}}{n !} .
$$

Obviously, the generating function of $B_{n, q}(\lambda)$ is

$$
\sum_{n=0}^{\infty} B_{n, q}(\lambda) \frac{t^{n}}{n !}=-t e^{\frac{t}{1-q}} \sum_{n=0}^{\infty} \frac{(-1)^{n} q^{n}}{1-\lambda q^{2 n}}\left(\frac{1}{1-q}\right)^{n} \frac{t^{n}}{n !} .
$$

Similarly, we can derive the following generating function of $E_{n, q}(x, \lambda)$ and $E_{n, q}(\lambda)$ by Definition 2.2 as below:

$$
\sum_{n=0}^{\infty} E_{n, q}(x, \lambda) \frac{t^{n}}{n !}=2 e^{\frac{t}{1-q}} \sum_{n=0}^{\infty} \frac{(-1)^{n} q^{(x+1) n}}{1+\lambda q^{2 n}}\left(\frac{1}{1-q}\right)^{n} \frac{t^{n}}{n !}
$$


and

$$
\sum_{n=0}^{\infty} E_{n, q}(\lambda) \frac{t^{n}}{n !}=2 e^{\frac{t}{1-q}} \sum_{n=0}^{\infty} \frac{(-1)^{n} q^{n}}{1+\lambda q^{2 n}}\left(\frac{1}{1-q}\right)^{n} \frac{t^{n}}{n !}
$$

respectively.

Clearly, it is easy to find the generating functions of $E_{n, q}(x)$ and $E_{n, q}$ by setting $\lambda=1$ in (3.2) and (3.3).

By (3.1), we calculate the following sum.

$$
\begin{aligned}
& \sum_{n=0}^{\infty}\left[[m]_{q}{ }^{n-1} \sum_{l=0}^{m-1} \lambda^{l} B_{n, q^{m}}\left(x+\frac{2 l+1-m}{m}, \lambda^{m}\right)\right] \frac{t^{n}}{n !} \\
& =\sum_{l=0}^{m-1} \frac{\lambda^{l}}{[m]_{q}} \sum_{n=0}^{\infty} B_{n, q^{m}}\left(x+\frac{2 l+1-m}{m}, \lambda^{m}\right) \frac{\left([m]_{q} t\right)^{n}}{n !} \\
& =-t e^{\frac{t}{1-q}} \sum_{n=0}^{\infty}\left(\frac{(-1)^{n} q^{(m x+1) n}}{1-\left(\lambda q^{2 n}\right)^{m}} \frac{1}{(1-q)^{n}}\right)\left(\sum_{l=0}^{m-1}\left(\lambda q^{2 n}\right)^{l}\right) \frac{t^{n}}{n !} \\
& =-t e^{\frac{t}{1-q}} \sum_{n=0}^{\infty} \frac{(-1)^{n} q^{(m x+1) n}}{1-\lambda q^{2 n}} \frac{1}{(1-q)^{n}} \frac{t^{n}}{n !} \\
& =\sum_{n=0}^{\infty} B_{n, q}(m x, \lambda) \frac{t^{n}}{n !} .
\end{aligned}
$$

Comparing the coefficient of $\frac{\mathrm{t}^{\mathrm{n}}}{\mathrm{n} !}$ on both sides of the above equation (3.4), we have Theorem 3.1.

Theorem 3.1. For a nonnegative integer $n$,

$$
B_{n, q}(m x, \lambda)=[m]_{q}^{n-1} \sum_{l=0}^{m-1} \lambda^{l} B_{n, q^{m}}\left(x+\frac{2 l+1-m}{m}, \lambda^{m}\right) .
$$

By the equations (3.1) and (3.2), we calculate the following sum.

$$
\begin{aligned}
& \sum_{n=0}^{\infty}\left[-\frac{n}{2}[m]_{q}^{n-1} \sum_{l=0}^{m-1} \lambda^{l} E_{n-1, q^{m}}\left(x+\frac{2 l+1-m}{m},-\lambda^{m}\right)\right] \frac{t^{n}}{n !} \\
& \quad=-t e^{\frac{t}{1-q}} \sum_{n=0}^{\infty} \frac{(-1)^{n} q^{(m x+1) n}}{1-\lambda q^{2 n}} \frac{1}{(1-q)^{n}} \frac{t^{n}}{n !}=\sum_{n=0}^{\infty} B_{n, q}(m x, \lambda) \frac{t^{n}}{n !} .
\end{aligned}
$$

Comparing the coefficient of $\frac{t^{n}}{n !}$ on both sides of (3.6), we obtain the result of the Theorem 3.2.

Theorem 3.2. For a nonnegative integer $n$,

$$
B_{n, q}(m x, \lambda)=-\frac{n}{2}[m]_{q}{ }^{n-1} \sum_{l=0}^{m-1} \lambda^{l} E_{n-1, q^{m}}\left(x+\frac{2 l+1-m}{m},-\lambda^{m}\right) .
$$

Using the results of the above two theorems, we get the following Corollary (3.3) immediately.

Corollary 3.3. For a nonnegative integer $n$,

$$
\sum_{l=0}^{m-1} \lambda^{l} B_{n, q^{m}}\left(x+\frac{2 l+1-m}{m}, \lambda^{m}\right)=-\frac{n}{2} \sum_{l=0}^{m-1} \lambda^{l} E_{n-1, q^{m}}\left(x+\frac{2 l+1-m}{m},-\lambda^{m}\right) .
$$


Letting $\mathrm{q} \rightarrow 1$ in the equations (3.5) and (3.7), we get the following.

Corollary 3.4. For a nonnegative integer $\mathrm{n}$ and letting $\mathrm{q} \rightarrow 1$,

$$
B_{n}(m x, \lambda)=\left\{\begin{array}{l}
m^{n-1} \sum_{l=0}^{m-1} \lambda^{l} B_{n}\left(x+\frac{2 l+1-m}{m}, \lambda^{m}\right), \\
-\frac{n}{2} m^{n-1} \sum_{l=0}^{m-1} \lambda^{l} E_{n-1}\left(x+\frac{2 l+1-m}{m},-\lambda^{m}\right) .
\end{array}\right.
$$

Putting $\lambda=1$ into the equations (3.5) and (3.7), we get the following.

Corollary 3.5. For a nonnegative integer $\mathrm{n}$ and $\lambda=1$,

$$
B_{n, q}(m x)=\left\{\begin{array}{l}
{[m]_{q}{ }^{n-1} \sum_{l=0}^{m-1} B_{n, q^{m}}\left(x+\frac{2 l+1-m}{m}\right)} \\
-\frac{n}{2}[m]_{q}{ }^{n-1} \sum_{l=0}^{m-1} E_{n-1, q^{m}}\left(x+\frac{2 l+1-m}{m}\right) .
\end{array}\right.
$$

Noticeably, when $\mathrm{q} \rightarrow 1$, we get the following.

Corollary 3.6. For a nonnegative integer $\mathrm{n}$, let $\mathrm{q} \rightarrow 1$ and $\lambda=1$,

$$
B_{n}(m x)=\left\{\begin{array}{l}
m^{n-1} \sum_{l=0}^{m-1} B_{n}\left(x+\frac{2 l+1-m}{m}\right) \\
-\frac{n}{2} m^{n-1} \sum_{l=0}^{m-1} E_{n-1}\left(x+\frac{2 l+1-m}{m}\right) .
\end{array}\right.
$$

\section{The q-Hurwitz-Lerch Zeta function}

The Hurwitz-Lerch zeta function $\Phi(z, s, a)$ is defined $([1,4,5,12]$, e.g., [2, pp.121, et seq.], [3]) as below:

$$
\Phi(z, s, a)=\sum_{n=0}^{\infty} \frac{z^{n}}{(n+a)^{s}}, \quad\left(a \in \mathbb{C} \backslash \mathbb{Z}_{0}^{-}, s \in \mathbb{C}, \text { when }|z|<1 \text { and } \mathfrak{R}(s)>1 \text { when }|z|=1\right) .
$$

$\Phi(z, s, a)$ contains, as its special cases, not only the Riemann and Hurwitz (or generalized) zeta functions:

$$
\zeta(s)=\Phi(1, s, 1)=\zeta(s, 1)=\frac{1}{2^{s}-1} \zeta\left(s, \frac{1}{2}\right)
$$

and

$$
\zeta(s, a)=\Phi(1, s, a)=\sum_{n=0}^{\infty} \frac{1}{(n+a)^{s}}, \quad\left(\Re(s)>1, a \notin \mathbb{Z}_{0}^{-}\right) .
$$

We first define the q-Hurwitz zeta function as follows:

Definition 4.1. The q-Hurwitz zeta function of the second kind is defined by

$$
\Phi_{\mathrm{q}}(z, \mathrm{~s}, \mathrm{a})=\sum_{\mathrm{n}=0}^{\infty} \frac{z^{\mathrm{n}}}{[2 \mathrm{n}+1+\mathrm{a}]_{\mathrm{q}}^{\mathrm{s}}}, \quad\left(\mathfrak{R}(\mathrm{a})>0, \mathrm{a} \in \mathbb{C} \backslash \mathbb{Z}_{0}^{-}\right) .
$$


We differentiate both side of (2.2) with respect to the variable $t$ which yields

$$
\left.\frac{d^{k}}{d t^{k}}\left(\sum_{n=0}^{\infty} B_{n, q}(a, \lambda) \frac{t^{n}}{n !}\right)\right|_{t=0}=\left.\frac{d^{k}}{d t^{k}}\left(-t \sum_{n=0}^{\infty} \lambda^{n} e^{[2 n+1+a]_{q} t}\right)\right|_{t=0} .
$$

The following equation can be obtained by summarizing the above equation

$$
B_{k, q}(a, \lambda)=-k \sum_{n=0}^{\infty} \frac{\lambda^{n}}{[2 n+1+a]_{q}^{1-k}}=-k \Phi_{q}(\lambda, 1-k, a) .
$$

Hence, we have the following relationship.

Theorem 4.2. For $n \in \mathbb{N}$ and $a \in \mathbb{C} \backslash \mathbb{Z}_{0}^{-}$,

$$
B_{n, q}(a, \lambda)=-n \Phi_{q}(\lambda, 1-n, a) .
$$

The above equation holds between the q-Apostol Bernoulli polynomials and the q-Hurwitz-Lerch zeta function of the second kind.

Letting $\mathrm{q} \rightarrow 1$ in (4.1), we get the following corollary.

Corollary 4.3. For $\mathrm{n} \in \mathbb{N}$, as $\mathrm{q} \rightarrow 1$ and $\mathrm{a} \in \mathbb{C} \backslash \mathbb{Z}_{0}^{-}$,

$$
B_{n}(a, \lambda)=-n \Phi(\lambda, 1-n, a) .
$$

The above equation holds between the Apostol Bernoulli polynomials and the Hurwitz-Lerch zeta function.

It follows that we define an analogue of the Hurwitz zeta function of the second kind as follows.

Definition 4.4. The L-function of the second kind is defined by

$$
L(s, a)=\sum_{n=0}^{\infty} \frac{1}{(2 n+1+a)^{s}}, \quad\left(\Re(s)>1, a \in \mathbb{C} \backslash \mathbb{Z}_{0}^{-}\right) .
$$

It follows that we define a q-analogue of the L-function of the second kind as follows.

Definition 4.5. The q-L-function of the second kind is defined by

$$
\mathrm{L}_{\mathrm{q}}(\mathrm{s}, \mathrm{a})=\sum_{n=0}^{\infty} \frac{1}{[2 \mathrm{n}+1+\mathrm{a}]_{\mathrm{q}}^{\mathrm{s}}}, \quad\left(\Re(s)>1, a \in \mathbb{C} \backslash \mathbb{Z}_{0}^{-}\right) .
$$

Obviously, $\Phi_{q}(1, s, a)=L_{q}(s, a)$. In the same way, we get the following.

Theorem 4.6. For $n \in \mathbb{N}$ and $a \in \mathbb{C} \backslash \mathbb{Z}_{0}^{-}$,

$$
B_{n, q}(a)=-n L_{q}(1-n, a) .
$$

The above equation holds between the q-Bernoulli polynomials and the q-L-function of the second kind.

Corollary 4.7. For $\mathrm{n} \in \mathbb{N}$ and $\mathrm{a} \in \mathbb{C} \backslash \mathbb{Z}_{0}^{-}$,

$$
B_{n}(a)=-n L(1-n, a) .
$$

The above equation holds between the Bernoulli polynomials and the q-L-function of the second kind. Finally, we introduce an analogue of Riemann zeta function of the second kind as follows. 
Definition 4.8. The l-function of the second kind is defined by

$$
l(s)=\sum_{n=1}^{\infty} \frac{1}{(2 n+1)^{s}}, \quad(\Re(s)>1) .
$$

It follows that we introduce a q-analogue of the l-function of the second kind.

Definition 4.9. The q-l-function is defined by

$$
\mathrm{l}_{\mathrm{q}}(\mathrm{s})=\sum_{\mathrm{n}=1}^{\infty} \frac{1}{[2 \mathrm{n}+1]_{\mathrm{q}}^{\mathrm{s}}}, \quad(\mathfrak{R}(\mathrm{s})>1) .
$$

Similarly, we have the following.

Theorem 4.10. For $n \in \mathbb{N}$,

$$
B_{n, q}=-n l_{q}(1-n) .
$$

The above equation holds between the q-Bernoulli numbers and the q-l-function of the second kind.

Corollary 4.11. For $n \in \mathbb{N}$,

$$
B_{n}=-n l(1-n) .
$$

The above equation holds between the Bernoulli numbers and the l-function of the second kind.

\section{Acknowledgment}

This work was supported by 2017 Hannam University Research Fund.

\section{References}

[1] T. M. Apostal, On the Lerch zeta function, Pacific J. Math., 1 (1951), 161-167. 4

[2] J. Choi, P. J. Anderson, H. M. Srivastava, Some q-extensions of the Apostol-Bernoulli and the Apostol-Euler polynomials of order $n$, and the multiple Hurwitz zeta function, Appl. Math. Comput., 199 (2008), 723-737. 4

[3] L. Comtet, Advanced combinatorics: the art of finite and infinite expansions, D. Reidel Publishing Co., Dordrecht/Boston, (1974). 1, 4

[4] T. Kim, Note on the Euler q-zeta functions, J. Number Theory, 129 (2009), 1798-1804. 4

[5] T. Kim, L.-C. Jang, H. K. Pak, A note on q-Euler and Genocchi numbers, Proc. Japan Acad. Ser. A Math. Sci., 77 (2001), 139-141. 4

[6] H. Y. Lee, N. S. Jung, C. S. Ryoo, A numerical investigation of the roots of the second kind $\lambda$-Bernoulli polynomials, Neural Parallel Sci. Comput., 19 (2011), 295-306. 1

[7] W. Magnus, F. Oberhettinger, R. P. Soni, Formulas and Theorems for the Special Functions of Mathematical Physics, Springer-verlag, New York, (1966). 1

[8] C. S. Ryoo, Analytic continuation of Euler polynomials and Euler zeta function, Discrete Dyn. Nat. Soc., 2014 (2014), 6 pages. 1

[9] C. S. Ryoo, Distribution of the roots of the second kind Bernoulli polynomials, J. Comput. Anal. Appl., 13 (2011), 971-976. 1

[10] J. Sándor, B. Crstici, Handbook of number theory II, Kluwer Academic Publishers, Dordrecht, (2004). 2.12

[11] Y. Simsek, On q-analogue of the twisted L-functions and q-twisted Bernoulli numbers, J. Korean Math. Soc., 40 (2003), 963-975. 1

[12] H. M. Srivastava, J. Choi, Series associated with the zeta and related functions, Kluwer Academic Publishers, Dordrecht, (2001). 4 